\title{
Pathological sequelae of long-haul COVID
}

\author{
Saurabh Mehandru ${ }^{1,2} \bowtie$ and Miriam Merad ${ }^{1,3 凶}$
}

\begin{abstract}
The world continues to contend with successive waves of coronavirus disease 2019 (COVID-19), fueled by the emergence of viral variants. At the same time, persistent, prolonged and often debilitating sequelae are increasingly recognized in convalescent individuals, named 'post-COVID-19 syndrome' or 'long-haul COVID'. Clinical symptomatology includes fatigue, malaise, dyspnea, defects in memory and concentration and a variety of neuropsychiatric syndromes as the major manifestations, and several organ systems can be involved. The underlying pathophysiological mechanisms are poorly understood at present. This Review details organ-specific sequelae of post-COVID-19 syndromes and examines the underlying pathophysiological mechanisms available so far, elaborating on persistent inflammation, induced autoimmunity and putative viral reservoirs. Finally, we propose diagnostic strategies to better understand this heterogeneous disorder that continues to afflict millions of people worldwide.
\end{abstract}

A of 15 November 2021, an estimated 254 million cases and 5.1 million deaths have been ascribed to COVID-19 ${ }^{1}$ As the world grapples with successive waves of infection fueled by the emergence of viral variants, in a subset of patients who recover, protracted disease symptoms, termed 'long COVID', 'long-haul COVID' or 'post-COVID syndrome', are being increasingly recognized. In the absence of a unifying disease definition, working constructs of long COVID have included the persistence of a constellation of symptoms for time periods varying from $>4$ weeks from symptom onset ${ }^{2}$ to symptoms that last for more than 3 months after onset ${ }^{3}$. Proposed guidelines from the National Institute for Health and Care Excellence, Scottish Intercollegiate Guidelines Network and the Royal College of General Practitioners, all in the UK, define 'acute COVID-19' as signs and symptoms of COVID-19 for up to 4 weeks; 'ongoing symptomatic COVID-19' as signs and symptoms of COVID-19 present from 4 weeks to 12 weeks of infection; and post-COVID-19 syndrome as signs and symptoms consistent with COVID-19 that are present for more than 12 weeks after infection and not attributable to alternative diagnoses ${ }^{4}$. The US Centers for Disease Control and Prevention uses the term 'post-COVID conditions' as an umbrella term for the wide range of health consequences that are present $\geq 4$ weeks after infection with severe acute respiratory syndrome coronavirus 2 (SARS-CoV-2).

Organ-specific sequelae. SARS-CoV-2, a novel $\beta$-coronavirus, is phylogenetically related to coronaviruses responsible for SARS and Middle East respiratory syndrome (MERS) (SARS-CoV and MERS-CoV, respectively). Notably, a subset of individuals who have had SARS or MERS are reported to demonstrate protracted neuropsychiatric symptoms ${ }^{5-9}$, sleep abnormalities ${ }^{9}$, persistent impairment of pulmonary function, including reductions in diffusion lung capacity $\left(D \mathrm{~L}_{\mathrm{CO}}\right)^{8,10-12}$, pulmonary fibrosis ${ }^{13}$, myalgias ${ }^{9}$ and functional disabilities such as reduced exercise tolerance ${ }^{8-12}$. Although aspects of long COVID are reminiscent of post-SARS and post-MERS syndromes, clinical and laboratory manifestations associated with long COVID appear to be protean, with the involvement of several organ systems.

The severity of acute COVID-19 has been linked to post-COVID-19 syndrome in one large nationwide study based on healthcase databases of the US Department of Veterans Affairs ${ }^{14}$. In another systematic review, although COVID-19-associated symptomatology was more pronounced in individuals with severe disease, individuals with mild and moderate disease also reported a wide range of symptoms after the resolution of clinical disease ${ }^{15}$. Finally, in a recent study of home-isolated, young (16to 30 -year-old) patients with COVID-19, nearly 60\% reported persistent symptoms at 6 months, independent of the severity of the initial illness ${ }^{16}$. Although multiple organ systems can be involved in acute COVID-19, the most common manifestations are systemic, respiratory, gastrointestinal, cardiovascular and neurological. By contrast, the most common manifestations of post-COVID-19 syndrome are systemic (fatigue and, poor concentration), neuropsychiatric (sleep abnormalities, chronic headache, 'brain fog', defects in memory, mood impairment and pain syndromes), cardiac (palpitations, syncope, dysrhythmias and postural symptoms) and respiratory (dyspnea and cough). Table 1 outlines the salient manifestations of acute COVID-19 and post-COVID-19 syndrome. Noteworthy clinical manifestations, summarized by specific organ systems, are detailed below, focusing mostly on patients with severe disease.

Given that lung involvement characterizes severe COVID-19, pulmonary symptoms including breathlessness ${ }^{17-20}$ and $\operatorname{cough}^{17,18}$ are noted in a substantial proportion of patients with long COVID and may ${ }^{21-24}$ or may not ${ }^{25}$ correlate with prior COVID-19 severity. Other lung-related manifestations included prolonged oxygen requirement ${ }^{18}$ and difficulty weaning off ventilators ${ }^{26}$, often related to the baseline pulmonary status. Persistent radiological abnormalities ${ }^{24,27-30}$ include ground-glass opacities, signs of reticulation, including course fibrous bands either with or without obvious parenchymal distortion, bronchiectasis and pulmonary fibrosis, and appeared to be related to greater severity of acute COVID-19 syndrome ${ }^{27,31}$. Abnormal lung function, including a reduction in $D \mathrm{~L}_{\mathrm{CO}}$, is the most commonly reported physiological impairment after acute COVID-19 and appears to be related to the severity of the acute illness ${ }^{27,28,30,32}$

A broad range of neurological and neuropsychiatric problems can cover acute COVID-19 syndrome, including olfactory and gustatory impairments, encephalopathy and delirium, stroke, neuromuscular complications, stress reactions and psychoses ${ }^{33}$. In addition to acute neuropsychiatric manifestations, COVID-19 may also produce protracted neurological and psychiatric sequelae in individuals with long COVID. Chronic malaise, sleep abnormalities, chronic headache and olfactory and gustatory impairments s, $20,27,31,33$

Precision Immunology Institute, Icahn School of Medicine at Mount Sinai, New York, NY, USA. ${ }^{2}$ Henry D. Janowitz Division of Gastroenterology, Department of Medicine, Icahn School of Medicine at Mount Sinai, New York, NY, USA. ${ }^{3}$ Department of Oncological Sciences, Icahn School of Medicine at Mount Sinai, New York, NY, USA. 凶e-mail: saurabh.mehandru@mssm.edu; miriam.merad@mssm.edu 
Table 1 | Summary of clinical manifestations during acute COVID-19 and post-COVID-19 syndrome

\begin{tabular}{|c|c|c|}
\hline & Acute COVID-19 (mild-severe) & Post-COVID-19 syndrome \\
\hline \multirow[t]{5}{*}{ Systemic manifestations } & Fever & Fatigue \\
\hline & Weakness & Poor concentration \\
\hline & Asthenia & Restriction of daily activities \\
\hline & Shivering & Chronic malaise \\
\hline & & Asthenia \\
\hline \multirow[t]{6}{*}{ Respiratory manifestations } & Dyspnea & Dyspnea \\
\hline & Cough & Persistent cough \\
\hline & Interstitial pneumonia & Exacerbation of asthma \\
\hline & ARDS & Reduced DLCO \\
\hline & Hemoptysis & Persistent radiological abnormalities \\
\hline & & Pleurisy \\
\hline \multirow[t]{16}{*}{ Neuropsychiatric manifestations } & Olfactory and gustatory impairments & Sleep abnormalities \\
\hline & Encephalopathy & Chronic headache \\
\hline & Delirium & Olfactory and gustatory impairments \\
\hline & Cerebrovascular accidents & Brain fog \\
\hline & Stress reactions & Defects in memory and concentration \\
\hline & Psychosis & Depression \\
\hline & Sensory loss & Anxiety \\
\hline & Gait abnormalities & Post-traumatic stress disorder \\
\hline & & Dizziness \\
\hline & & Imbalance \\
\hline & & Vertigo \\
\hline & & Psychosis and hallucinations \\
\hline & & Small fiber neuropathy \\
\hline & & Postural tremor \\
\hline & & Pain syndromes \\
\hline & & Neurodegenerative disorders \\
\hline \multirow[t]{3}{*}{ Musculo-skeletal manifestations } & Myalgia & Myalgia \\
\hline & Joint pain & Joint pain \\
\hline & & Small joint arthritis \\
\hline \multirow[t]{8}{*}{ Cardiac manifestations } & Myocardial injury & Atypical chest pain \\
\hline & Myocarditis & Chest tightness \\
\hline & Acute heart failure & Palpitations \\
\hline & Cardiomyopathy & Tachycardia \\
\hline & Cardiac dysrhythmias & Conduction abnormalities and dysrhythmias \\
\hline & & Orthostatic hypotension \\
\hline & & Vasovagal syncope \\
\hline & & Postural orthostatic tachycardia syndrome \\
\hline \multirow[t]{2}{*}{ Vascular manifestations } & Diffuse intravascular coagulation & Phlebitis and thrombophlebitis \\
\hline & Thromboembolic events & \\
\hline \multirow[t]{7}{*}{ Gastrointestinal manifestations } & Diarrhea & Loss of appetite \\
\hline & Nausea & Abdominal pain \\
\hline & Vomiting & Nausea \\
\hline & Abdominal pain & Weight loss \\
\hline & Abnormal liver function tests & Altered bowel motility \\
\hline & & Irritable bowel syndrome \\
\hline & & Dysphagia \\
\hline Endocrine manifestations & Diabetes mellitus & Persistent glycemic abnormalities \\
\hline
\end{tabular}


Table 1 | Summary of clinical manifestations during acute COVID-19 and post-COVID-19 syndrome (continued)

\begin{tabular}{|c|c|c|}
\hline & Acute COVID-19 (mild-severe) & Post-COVID-19 syndrome \\
\hline & Diabetic ketoacidosis & Subacute thyrotoxicosis \\
\hline & & Hashimoto's thyroiditis \\
\hline \multirow[t]{2}{*}{ Renal manifestations } & Acute kidney injury & Decreased GFR \\
\hline & & Microhematuria \\
\hline \multirow{2}{*}{ Dermatological manifestations } & & Telogen effluvium \\
\hline & & Nail alterations \\
\hline \multirow[t]{3}{*}{ Miscellaneous manifestations } & Conjunctivitis & Hearing loss \\
\hline & Sore throat & Tinnitus \\
\hline & Neck pain & Red eyes \\
\hline \multirow{9}{*}{ Lab abnormalities } & & MMP-7 \\
\hline & & HGF \\
\hline & & Elevated D-dimer \\
\hline & & Anemia \\
\hline & & Lipid abnormalities \\
\hline & & Elevated $\mathrm{HbA} 1 \mathrm{C}$ \\
\hline & & Reduced serum albumin \\
\hline & & Abnormal liver function tests \\
\hline & & Thrombocytosis \\
\hline
\end{tabular}

ARDS, acute respiratory distress syndrome; DLCO, diffusing capacity for carbon monoxide; GFR, glomerular filtration rate; HGF, hepatocyte growth factor; HbA1c, hemoglobin A1C; LCN-2, lipocalin 2; MMP-7, matrix metalloprotease 7.

are commonly reported. In addition, neurodegenerative disorders such as Parkinson's disease ${ }^{34}$ and cognitive impairment ranging from brain fog to the acceleration of Alzheimer's disease ${ }^{34,35}$ are described in patients who have had acute COVID. A range of psychiatric disorders, including a first psychiatric diagnosis of conditions such as depression, anxiety and post-traumatic stress disorder (PTSD), are reported in patients with post-COVID syndrome ${ }^{36}$.

Myocardial injury, myocarditis, acute heart failure, cardiomyopathy and cardiac dysrhythmias have all been reported during the acute phase of COVID-1937. Patients who have had acute or long COVID report chest pain, chest tightness and ongoing palpitations ${ }^{17,27,38,39}$. Cardiovascular magnetic resonance imaging (cMRI) performed in a cohort of 100 patients with COVID-19 71 (median; range 64-92) days after diagnosis identified cardiac involvement in $78 \%$ of patients and ongoing myocardial inflammation in $60 \%$ of patients, independent of pre-existing conditions, severity and overall course of the acute illness ${ }^{40}$. In another cohort of 26 athletes with mild acute COVID, cMRI performed 11-53 days after diagnosis revealed myocarditis in $15 \%$ and evidence of prior myocardial injury in $31 \%$ of the individuals ${ }^{41}$. Evidence of adverse ventricular remodeling was noted in about $30 \%$ of patients 3 months after discharge from hospitalization with COVID-19-associated pneumonia $^{42}$, suggesting cardiac sequelae.

Thrombotic complications, including diffuse intravascular coagulation and thromboembolic events involving different organs, are well recognized during acute COVID-19, and owing to which anti-thrombotic therapies are utilized in patient with severe disease ${ }^{43}$. However, the rate of venous thromboembolism in the post-acute COVID-19 setting is considered to be $<5 \%{ }^{44}$, with the caveat that these data are limited by small sample size and inadequate follow-up. Currently, in ambulatory patients with COVID-19, or those after discharge, routine administration of thromboprophylaxis is not recommended ${ }^{45}$. However, owing to the potential for persistent hypercoagulability post-COVID, high-quality data from randomized controlled trials are needed.

Given the high expression of angiotensin-converting enzyme 2 (ACE2) on the brush border of the small intestinal mucosa ${ }^{46}$, gastrointestinal symptoms, including diarrhea, nausea, vomiting and abdominal pain, are reported in $15-50 \%$ of patients ${ }^{47,48}$, and abnormal liver function tests in $20 \%{ }^{48}$ of patients during acute COVID- 19. Patients with long COVID report a range of gastrointestinal symptoms including loss of appetite, abdominal pain, nausea, weight loss, altered bowel motility patterns and new or exacerbated irritable bowel syndrome ${ }^{49}$. In addition, prolonged fecal shedding of SARS-CoV-2 (ref. ${ }^{50}$ ) and the presence of virions in intestinal enterocytes ${ }^{51}$ have been reported, as discussed in detail below.

Owing to the expression of ACE2 by pancreatic islet cells, the SARS coronavirus was found to cause islet cell injury and result in acute type 1 diabetes mellitus ${ }^{52}$. New-onset diabetes and diabetic ketoacidosis have been reported in patients with acute COVID-19 (ref. ${ }^{53}$ ) through both direct and indirect mechanisms ${ }^{54}$. Importantly, patients who recovered from COVID-19 also showed significantly 
higher fasting insulin levels, C-peptide levels, homeostasis model assessment of $\beta$-cell dysfunction and homeostasis model assessment of insulin resistance compared with healthy controls, with no differences observed in the insulin:proinsulin ratio, resulting in persistent glycemic abnormalities in $35 \%$ of 551 patients for at least 2 months after COVID-19 (ref. ${ }^{54}$ ). Among other endocrine abnormalities, subacute thyrotoxicosis ${ }^{55}$, Hashimoto's thyroiditis ${ }^{56}$ and Graves' disease $^{57}$ have all been reported weeks after the resolution of respiratory symptoms in patients with COVID-19.

Up to one-third of critically ill patients with COVID-19 that require mechanical ventilation may develop acute kidney injury requiring renal replacement therapy, which is associated with high mortality ${ }^{58,59}$. However, among these patients, the rate of renal recovery was noted to be high in one study ${ }^{59}$, whereas a different study reported decreased glomerular filtration rate in $35 \%$ of patients 6 months after acute COVID ${ }^{27}$.

A large international registry from the American Academy of Dermatology and International League of Dermatological Societies reported 716 cases of new-onset dermatological symptoms in patients with confirmed/suspected COVID-19 (ref. $\left.{ }^{60}\right)$. Of the 171 patients in the registry with laboratory-confirmed COVID-19, the most common morphologies were morbilliform (22\%), pernio like (18\%), urticarial (16\%), macular erythema (13\%), vesicular (11\%), papulosquamous $(9.9 \%)$ and retiform purpura $(6.4 \%)^{60}$. Thus, multiple cutaneous manifestations are associated with acute COVID-19. A minority of patients (3\%) complained of a skin $\operatorname{rash}^{27}$ at the 6-month follow-up after acute COVID-19, although up to $20 \%$ of patients may complain of persistent hair loss ${ }^{20,27}$. Hair shedding has also been reported in patients around 40-50 d after COVID infections. Acute telogen effluvium, a self-limiting cause of diffuse hair shedding, is known to occur in the $2-3$ months, and to resolve in the 3-6 months, after a triggering event that can include an inflammatory state ${ }^{61}$. In the post-COVID setting, acute telogen effluvium seems to appear and resolves sooner than commonly described acute telogen effluvium ${ }^{62}$. Factors such as hypoxia, inflammation (specifically interleukin (IL)-6) and medications have a role in the development and severity of telogen effluvium ${ }^{62}$.

Pathophysiology. Mild COVID-19 is associated with a potent initial innate antiviral response induction and viral neutralization, culminating in less immunopathology and clinical resolution of disease ${ }^{63}$. In severe COVID-19, on the other hand, uncontrolled replication of SARS-CoV evades the host innate immune activation during its initial steps, with a consequent increase in proinflammatory responses and immune cell infiltration (including recruitment of inflammatory monocytes, neutrophils and activated $\mathrm{T}$ cells) to the lungs, which provokes tissue damage ${ }^{64}$. Aberrant inflammatory responses characterized by increased production of potent inflammatory cytokines, including tumor necrosis factor (TNF) and IL-6, further stimulate downstream immunopathological events, activation of the coagulation cascade and multiorgan involvement. These pathways are reviewed elsehwere ${ }^{65}$. The immunological 'bridge' between acute COVID-19 and post-COVID-19 syndrome has been elusive. Below, we summarize the available data and encapsulate the known and hypothesized pathophysiology of the post-COVID syndrome

An important consideration while unraveling the pathophysiology of the post-COVID syndrome is a careful annotation of the clinical symptomatology. Distinguishing lingering symptoms of acute disease site from new symptoms that appear after resolution of the acute disease may help distinguish between potential disease drivers. Confounding variables may, however, include components of PTSD that may blur patients' own evaluation of clinical symptoms and require careful neuropsychiatric evaluations.

There are many available hypotheses that could explain persistence of clinical symptoms, as summarized in Fig. 1 . The severity of the inflammatory response to new infectious threats may lead to delay or defects in the resolution of inflammation, which may explain the persistence of symptoms, especially at infectious sites such as the lung or upper respiratory tracts, and their systemic nature. A recent study prospectively examined a cohort of 31 individuals with long-haul COVID (based on the presence of one of three major symptoms: dyspnea, fatigue and chest pain), age and gender matched with 31 individuals who had prior COVID infection but lacked long-haul COVID symptoms. In addition, 25 individuals infected with common cold coronaviruses and unexposed healthy individuals $(n=46)$ served as controls ${ }^{66}$. Patients with long-haul COVID demonstrated persistent increases in the frequency of activated $\mathrm{CD} 14^{+} \mathrm{CD} 16^{+}$monocytes and plasmacytoid dendritic cells, compared with control individuals 8 months after infection $^{66}$. Furthermore, patients with long-haul COVID demonstrated persistent elevation in the levels of type I (IFN $\beta$ ) and type III (IFN $\lambda 1$ ) interferon 8 months post-infection ${ }^{66}$. In the study, a combination of IFN $\beta$, pentraxin 3 , IFN $\gamma$, IFN $\lambda 2 / 3$ and IL- 6 was associated with long-haul COVID, with an accuracy ranging from $78.5 \%$ to $81.6 \%{ }^{66}$. These analytes have been associated with acute severe disease, suggesting a delayed or defective resolution of inflammation in long-haul COVID individuals. On the basis of our own studies, a delay in the resolution of inflammation is likely to be quite common among patients with symptomatic diseases, and lingering symptoms wane around 6 months post-infection (unpublished data). At the opposite end of the spectrum hyperinflammatory responses can lead to irreversible lung fibrosis that severely compromises respiratory effector function. Notably, although subjective symptoms such as fatigue and dyspnea do not track with the severity of inflammation, objective markers associated with persistent neutrophil activation (lipocalin-2), fibrosis signaling (matrix metalloprotease-7) and alveolar epithelial repair (hepatocyte growth factor) strongly associate with the severity of inflammation and impaired pulmonary function test ${ }^{25}$. The development of persistent lung injuries may reflect the ability of SARS-CoV-2 to alter mechanisms of immune homeostasis that are key modulators of tissue inflammation. In lung tissue, resident macrophages (also known as alveolar macrophages) are a subset of macrophages that self-renew locally independent of circulating monocytes ${ }^{67}$. Alveolar macrophages have a key role in the clearance of surfactant and damaged cells with a crucial involvement in the maintenance of lung tissue integrity. Alveolar macrophages are strongly reduced in the bronchoalveolar lavage of patients with severe COVID-19 (ref. ${ }^{68}$ ) where they are replaced by inflammatory monocytes. The differentiation, survival and local maintenance of tissue-resident macrophages are dependent on the production of CSF-2 by alveolar type II (AT2) lung epithelial cells $^{69}$. AT2 cells express ACE2 receptors ${ }^{70}$ and are a key target of the SARS-CoV-2 virus ${ }^{71}$. It is possible that damage to AT2 in COVID-19 contributes to an early reduction of the tissue-resident macrophage compartment, thus altering an important immune homeostatic compartment of the lung tissue. Recent single-cell analyses of lungs from individuals with fatal COVID-19 revealed the adoption of an inflammation-associated transient progenitor cell state by AT2 cells that failed to undergo full transition into AT1 cells, resulting in impaired lung regeneration ${ }^{72}$. Furthermore, a subset of pathological, CTHRC1-expressing fibroblasts ${ }^{73}$ was identified in the lungs that potentially contribute to rapidly ensuing pulmonary fibrosis in COVID-19 (ref. ${ }^{72}$ ). This fibrotic state is probably driven by transforming growth factor- $\beta^{74}$ and epithelium-derived IL-6 (ref. ${ }^{72}$ ).

To help boost the homeostatic function of tissue-resident macrophages, a clinical trial was initiated to measure the clinical benefit of CSF-2 treatment in patients with severe COVID-19. The results of this trial have not been published yet, but it might be difficult to obtain clear clinical benefit given the potential ability of CSF-2to enhance the survival and activity of inflammatory monocyte-derived macrophages, which have an important role in the damaging inflammatory responses. This condition highlights 


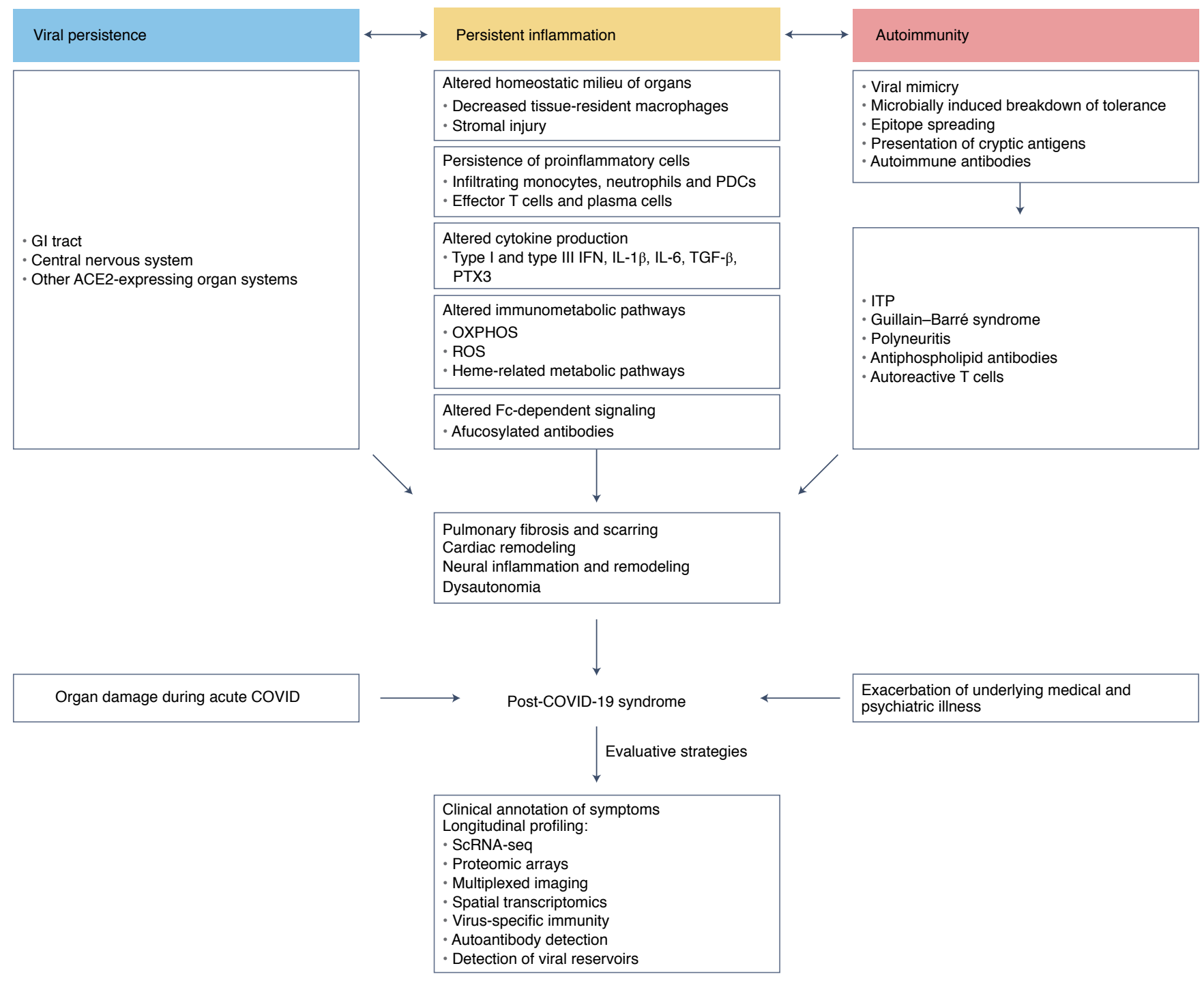

Fig. 1 | Putative mechanisms and diagnostic strategies for patients with post-COVID-19 syndromes. Delayed resolution of inflammation, autoimmunity and viral persistence represents overlapping mechanisms that may contribute to the pathogenesis of post-COVID-19 syndromes. Strategies to better characterize patients with post-COVID-19 syndromes are indicated. ITP, idiopathic thrombocytopenic purpura; OXPHOS, oxidative phosphorylation; PDCs, plasmacytoid dendritic cells; ROS, reactive oxygen species; TGF- $\beta$, transforming growth factor- $\beta$.

the need to uncouple homeostatic from damaging immunity, and to develop targeted therapies that may boost tissue-resident macrophage immune and homeostatic function, while limiting the damaging function of monocyte-derived macrophages.

Acute COVID-19 is associated with a prothrombotic state ${ }^{75}$ that can lead to endothelial injury ${ }^{76}$ and platelet activation ${ }^{77}$, driven directly and indirectly by immune cell activation ${ }^{78}$. Although the lungs bear the brunt of thrombotic damage ${ }^{76}$, several organopathies are linked to thrombotic sequelae of SARS-CoV-2 infection, including cardiovascular ${ }^{27,79}$, neurological ${ }^{80}$ and renal ${ }^{81}$ injuries. At present, the duration of this prothrombotic state, and potentially its extension into the post-COVID-19 syndrome, are unclear.

Persistent elevation of proinflammatory cytokines such as IL-6, IL-1 $\beta$ and TNF may have multiple systemic as well as organ-specific effects that could potentially be associated with manifestations of the post-COVID-19 syndrome. These include altered cardiac remodeling ${ }^{82}$, cardiac dysrhythmias ${ }^{83}$, neuroinflammation ${ }^{84}$, neurodegeneration ${ }^{85}$, renal injury ${ }^{86}$, peripheral insulin resistance ${ }^{87}$, bone resorption ${ }^{88}$ and hair loss ${ }^{27}$. Therefore, objective quantification of proinflammatory cytokines may help to identify patients with persistent systemic inflammation as a cause of post-COVID-19 syndromic manifestations.

Finally, a recent study, reporting on 'immunological recovery' after COVID-19, noted persistent immunological abnormalities, including increased frequencies of effector $\mathrm{T}$ and $\mathrm{B}$ cells, and activation of oxidative phosphorylation-, reactive oxygen speciesand heme-related metabolic pathways ${ }^{63}$. These abnormalities were speculated to contribute to post-COVID-19 syndrome, although no direct evidence was available from the present study ${ }^{63}$.

Macrophages can detect viruses directly through Toll-like receptors $^{89}$ or through immune complexes via Fc receptors ${ }^{90}$. The recognition of immune complexes triggers macrophage activation and the release of effector molecules that contribute to viral clearance. The rapid clearance of immune complexes is critical for immune homeostasis and the resolution of inflammation ${ }^{90}$. There is significant functional heterogeneity among people in the Fc-receptor signaling axis. Recent studies have revealed specific modification of Fc domains, characterized by the absence of a core fucose residue (afucosylation), in people with severe COVID-19 and in a group of patients with mild disease who later progressed to more severe 
symptoms ${ }^{91}$. It is possible that afucosylated SARS-CoV-2 antibodies can also contribute to the imbalanced inflammatory response in patients with post-COVID syndrome and measuring the levels of afucosylated immunoglobulin $\mathrm{G}$ antibodies may help identify patients at risk of post-COVID syndrome.

Another immune dysregulatory response that may contribute to post-COVID lingering symptoms is the development of a damaging, self-sustaining, autoimmune response against self-tissue antigens that persists after the virus has been eliminated. The induction of autoimmunity after a number of viral infections, including parvovirus B19, Epstein-Barr virus, cytomegalovirus, herpes virus 6, human $\mathrm{T}$ cell lymphotropic virus type 1 , hepatitis $\mathrm{A}$ and $\mathrm{C}$ virus and rubella virus, has been widely described ${ }^{92}$. Proposed mechanisms include viral mimicry ${ }^{93}$, breakdown of tolerance against self-antigens presented in the context of microbial infection ${ }^{94}$ and epitope spreading, as well as presentation of cryptic antigens ${ }^{95}$. A number of autoimmune diseases and syndromes have been associated with COVID-19 thus far. These include idiopathic thrombocytopenic purpura ${ }^{96}$, Guillain-Barré syndrome ${ }^{97}$, Miller Fisher syndrome and polyneuritis cranialis ${ }^{98}$, antiphospholipid antibodies and lupus anticoagulant ${ }^{99}$, Kawasaki-like disease and multisystem inflammatory syndrome in children ${ }^{100}$. Several studies have reported the presence of autoantibodies in patients with COVID-19 (refs. ${ }^{101-103}$ ). However, the prevalence of autoantibodies in patients with post-COVID-19 syndromes and in the general healthy population has not been well studied, and the development of assays to measure the contribution of these autoantibodies to tissue damage is still needed. In addition to autoantibodies, it is also possible that autoreactive $\mathrm{T}$ cells contribute to tissue damage and post-COVID syndrome. There has been little effort to measure cellular immunity in COVID-19 and the detection of self-reactive clones may help to shed light on the pathophysiology of a subset of patients with post-COVID syndrome.

Reports of improved clinical symptoms on administration of anti-SARS-CoV-2 vaccines in patients with post-COVID syndrome ${ }^{104}$ have led to suggestions of a viral reservoir in patients with post-COVID syndrome. Furthermore, in patients who have recovered from COVID-19, the memory B cell pool continues to display clonal turnover even after 6 months of infection ${ }^{51}$, and the antibodies that they express have greater somatic hypermutation, resistance to receptor-binding domain mutations and increased potency, indicative of continued evolution of the humoral response in a manner that is consistent with antigen persistence ${ }^{51}$.

In addition to the lungs and nasopharynx, SARS-CoV-2 replicates in ACE2-expressing cells in the small intestine ${ }^{47}$. Furthermore, viral RNA has been detected in stool samples even after the virus has been cleared from the nasopharyn $\mathrm{x}^{105}$. To determine whether there might be antigen persistence in the intestine after resolution of clinical illness, we obtained biopsies from the gastrointestinal tract of 14 individuals at an average of 4 months (range 2.8-5.7 months) after initial COVID-19 diagnosis ${ }^{51}$. SARS-CoV-2 $\mathrm{N}$ protein was detected in intestinal enterocytes in 5 out of 14 individuals ${ }^{51}$. Furthermore, 3 out of the 14 participants produced PCR amplicons that were sequence verified as SARS-CoV-2 (ref. ${ }^{51}$ ). Viral detection in the intestinal epithelium was patchy and sporadic, and none of the patients had symptoms of long COVID. Human body fluids, including bronchoalveolar lavage, sputum, saliva, blood, urine and feces, have also been demonstrated to harbor the virus ${ }^{106}$.

These data provide proof of principle that SARS-CoV-2 can potentially persist in specific tissues in a manner that would be consistent with the persistence of other nonretroviral RNA viruses ${ }^{107}$. However, much remains to be determined about SARS-CoV-2 persistence in humans. For example, it is unclear whether tissues such as the intestines provide a survival advantage to the virus. In addition, the role of the microbiome in viral pathogenesis and persistence remains undetermined. Furthermore, the relationship between intestinal viral persistence and long COVID symptoms remains to be studied. Local and repetitive measurements of viral proteins, as well as detailed imaging studies, could help identify viral reservoirs and measure their eradication after vaccination.

High-dimensional profiling of the systemic and local immunity of patients with COVID-19. The wide range of symptoms and likely pathophysiology of patients with post-COVID syndrome strongly highlight the need for careful annotation of clinical symptomatology and unbiased profiling of the immune system of patients. Optimal profiling of the immune response before and at different times during the post-COVID syndrome should best help unravel the pathophysiological mechanisms that contribute to the development of the post-COVID syndrome (Fig. 1). Unbiased profiling should include the measurement of systemic and local secreted inflammatory proteins, single-cell RNA profiling of circulating and local immune cells that accumulate at the disease site, as well as detailed ex vivo imaging of diseased organs. Multiplex imaging and spatial transcriptomics analysis of tissue biopsies are also very helpful for measuring the extent of the tissue damage, as well as cellular interactions of immune cells, inflammatory molecules and damaged cells and the potential presence of a viral reservoir. Detailed analysis of humoral and cellular viral-specific and self-specific immunity, as well as their accumulation at the disease site, can help parse the contribution of autoimmune diseases. These results should be compared with similar measurements performed in age- and comorbidities-matched but otherwise healthy individuals. Additional controls could include patients with other viral infections and patients with autoinflammatory diseases. These control cohorts are critical because there are few data on the homeostatic levels of immune molecules across the general population, including in patients with comorbidities. This is particularly relevant because, for example, patients with chronic kidney disease ${ }^{108}$ or obesity $^{109}$, who are at increased risk of severe COVID-19, have high levels of inflammatory molecules in the blood circulation, which in the absence of proper control can be mistakenly diagnosed as virus-driven inflammation. Furthermore, other variables, including COVID-19 vaccine status, need to be considered when interpreting the data. Profiling of disease sites should include, whenever possible, objective metrics of tissue damage as well as high-dimensional profiling of the inflamed organ. Together, the use of these complementary approaches could help unravel the cellular source of inflammation and foster the understanding of disease pathophysiology and the development of new therapeutic targets.

For example, using proteomics profiling, we have found that high systemic levels of S100A12 strongly correlated with disease severity, whereas single-cell RNA sequencing and multiplex imaging revealed that circulating monocytes and neutrophils, but not tissue-resident macrophages, are the main source of S100A12 (Chen, S. T. and M.M. unpublished data). These results suggest that misguided systemic myeloid response may contribute to the pathophysiology of acute COVID-19 and potentially also to the post-COVID syndrome, and emphasize the knowledge gained by the integration of different immune datasets.

\section{Conclusions and perspectives}

A notable proportion of patients with COVID-19 demonstrate residual symptoms beyond acute infection. In a subset of these individuals, the symptoms may persist beyond 12 weeks of disease onset and are classified as post-COVID-19 syndrome. Multiple organs may be involved, but the dominant manifestations of post-COVID-19 syndrome are pulmonary, neuropsychiatric, cardiovascular and gastrointestinal. Owing to the heterogeneity of disease pathogenesis and manifestations, there is a crucial need to institute canonical disease definitions that are based on objective clinical and laboratory criteria. Rigorous high-dimensional, serial 
profiling of tissues and peripheral blood, linking pathophysiological disruptions to clinical presentations and outcomes, will help delineate what are likely to be multiple syndromes that are still encapsulated in the term post-COVID-19 syndrome. In February 2021, the National Institutes of Health launched a major initiative known as the PASC initiative to bring together clinicians and researchers to study how to prevent and treat the long-term effects of SARS-CoV-2 infection. One major objective is to collect data from patients who have had COVID-19, both with and without long-term symptoms, and compare them with people who may never have been infected by SARS-CoV-2. The initiative will help to collect large patient cohorts, including children and adults from diverse backgrounds, to ensure that the findings apply to the communities that have been most affected by COVID-19. Here again, it will be important to use cutting-edge technologies to decipher the molecular heterogeneity of post-COVID syndrome and identify precise druggable targets tailored to these defects.

Received: 25 August 2021; Accepted: 30 November 2021; Published online: 1 February 2022

\section{References}

1. Dong, E., Du, H. \& Gardner, L. An interactive web-based dashboard to track COVID-19 in real time. Lancet Infect. Dis. 20, 533-534 (2020).

2. Nalbandian, A. et al. Post-acute COVID-19 syndrome. Nat. Med. 27, 601-615 (2021).

3. Venkatesan, P. NICE guideline on long COVID. Lancet Respir. Med. 9, 129 (2021).

4. Shah, W., Hillman, T., Playford, E. D. \& Hishmeh, L. Managing the long term effects of covid-19: summary of NICE, SIGN, and RCGP rapid guideline. BMJ 372, n136 (2021).

5. Lam, M. H. et al. Mental morbidities and chronic fatigue in severe acute respiratory syndrome survivors: long-term follow-up. Arch. Intern. Med. 169, 2142-2147 (2009)

6. Lee, S. H. et al. Depression as a mediator of chronic fatigue and post-traumatic stress symptoms in Middle East respiratory syndrome survivors. Psychiatry Invest. 16, 59-64 (2019).

7. Rogers, J. P. et al. Psychiatric and neuropsychiatric presentations associated with severe coronavirus infections: a systematic review and meta-analysis with comparison to the COVID-19 pandemic. Lancet Psychiatry 7, 611-627 (2020).

8. Ahmed, H. et al. Long-term clinical outcomes in survivors of severe acute respiratory syndrome and Middle East respiratory syndrome coronavirus outbreaks after hospitalisation or ICU admission: a systematic review and meta-analysis. J. Rehabil. Med. 52, jrm00063 (2020).

9. Moldofsky, H. \& Patcai, J. Chronic widespread musculoskeletal pain, fatigue, depression and disordered sleep in chronic post-SARS syndrome: a case-controlled study. BMC Neurol. 11, 37 (2011).

10. Ngai, J. C. et al. The long-term impact of severe acute respiratory syndrome on pulmonary function, exercise capacity and health status. Respirology 15, 543-550 (2010).

11. Hui, D. S. et al. Impact of severe acute respiratory syndrome (SARS) on pulmonary function, functional capacity and quality of life in a cohort of survivors. Thorax 60, 401-409 (2005).

12. Ong, K. C. et al. Pulmonary function and exercise capacity in survivors of severe acute respiratory syndrome. Eur. Respir. J. 24, 436-442 (2004).

13. Das, K. M. et al. Follow-up chest radiographic findings in patients with MERS-CoV after recovery. Indian J. Radio. Imaging 27, 342-349 (2017).

14. Al-Aly, Z., Xie, Y. \& Bowe, B. High-dimensional characterization of post-acute sequelae of COVID-19. Nature 594, 259-264 (2021).

15. Salamanna, F, Veronesi, F, Martini, L., Landini, M. P. \& Fini, M. Post-COVID-19 syndrome: the persistent symptoms at the post-viral stage of the disease. A systematic review of the current data. Front. Med. 8, 653516 (2021)

16. Blomberg, B. et al. Long COVID in a prospective cohort of home-isolated patients. Nat. Med. 27, 1607-1613 (2021)

17. Carfi, A., Bernabei, R., Landi, F. G. \& Against COVID-19 post-acute care study group. Persistent symptoms in patients after acute COVID-19. JAMA 324, 603-605 (2020)

18. Chopra, V., Flanders, S. A., O’Malley, M., Malani, A. N. \& Prescott, H. C. Sixty-day outcomes among patients hospitalized with COVID-19. Ann. Intern. Med. 174, 576-578 (2021).

19. Halpin, S. J. et al. Postdischarge symptoms and rehabilitation needs in survivors of COVID-19 infection: a cross-sectional evaluation. J. Med. Virol. 93, 1013-1022 (2021).
20. Garrigues, E. et al. Post-discharge persistent symptoms and health-related quality of life after hospitalization for COVID-19. J. Infect. 81, e4-e6 (2020).

21. Han, X. et al. Six-month follow-up chest CT findings after severe COVID-19 pneumonia. Radiology 299, E177-E186 (2021).

22. Truffaut, L. et al. Post-discharge critical COVID-19 lung function related to severity of radiologic lung involvement at admission. Respir. Res. 22, 29 (2021).

23. Weerahandi, $\mathrm{H}$. et al. Post-discharge health status and symptoms in patients with severe COVID-19. J. Gen. Intern. Med. 36, 738-745 (2021).

24. van Gassel, R. J. J. et al. High prevalence of pulmonary sequelae at 3 months after hospital discharge in mechanically ventilated survivors of COVID-19. Am. J. Respir. Crit. Care Med. 203, 371-374 (2021).

25. Chun, H. J. et al. Immunofibrotic drivers of impaired lung function in postacute sequelae of SARS-CoV-2 infection. JCI Insight https://doi.org/ 10.1172/jci.insight.148476 (2021)

26. Martin-Villares, C., Perez Molina-Ramirez, C., Bartolome-Benito, M., Bernal-Sprekelsen, M., \& COVID ORL ESP Collaborative Group. Outcome of 1890 tracheostomies for critical COVID-19 patients: a national cohort study in Spain. Eur. Arch. Otorhinolaryngol. 278, 1605-1612 (2021).

27. Huang, C. et al. 6-month consequences of COVID-19 in patients discharged from hospital: a cohort study. Lancet 397, 220-232 (2021).

28. Zhao, Y.-m. et al. Follow-up study of the pulmonary function and related physiological characteristics of COVID-19 survivors three months after recovery. Eclinicalmedicine 25, 100463 (2020).

29. Shah, A. S. et al. A prospective study of 12 -week respiratory outcomes in COVID-19-related hospitalisations. Thorax 76, 402-404 (2021).

30. Shaw, B., Daskareh, M. \& Gholamrezanezhad, A. The lingering manifestations of COVID-19 during and after convalescence: update on long-term pulmonary consequences of coronavirus disease 2019 (COVID-19). Radiol. Med. 126, 40-46 (2021).

31. Arnold, D. T. et al. Patient outcomes after hospitalisation with COVID-19 and implications for follow-up: results from a prospective UK cohort. Thorax 76, 399-401 (2021)

32. Mendez, R. et al. Reduced diffusion capacity in COVID-19 survivors. Ann. Am. Thorac. Soc. 18, 1253-1255 (2021)

33. Manolis, T. A., Apostolopoulos, E. J., Manolis, A. A., Melita, H. \& Manolis, A. S. COVID-19 infection: a neuropsychiatric perspective. J. Neuropsychiatry Clin. Neurosci. 33, 266-279 (2021).

34. Ferini-Strambi, L. \& Salsone, M. COVID-19 and neurological disorders: are neurodegenerative or neuroimmunological diseases more vulnerable? J. Neurol. 268, 409-419 (2021)

35. Heneka, M. T., Golenbock, D., Latz, E., Morgan, D. \& Brown, R. Immediate and long-term consequences of COVID-19 infections for the development of neurological disease. Alzheimers Res. Ther. 12, 69 (2020).

36. Taquet, M., Luciano, S., Geddes, J. R. \& Harrison, P. J. Bidirectional associations between COVID-19 and psychiatric disorder: retrospective cohort studies of 62354 COVID-19 cases in the USA. Lancet Psychiatry 8, 130-140 (2021).

37. Long, B., Brady, W. J., Koyfman, A. \& Gottlieb, M. Cardiovascular complications in COVID-19. Am. J. Emerg. Med. 38, 1504-1507 (2020).

38. Carvalho-Schneider, C. et al. Follow-up of adults with noncritical COVID-19 two months after symptom onset. Clin. Microbiol. Infect. 27, 258-263 (2021).

39. Dennis, A. et al. Multiorgan impairment in low-risk individuals with post-COVID-19 syndrome: a prospective, community-based study. BMJ Open 11, e048391 (2021).

40. Puntmann, V. O. et al. Outcomes of cardiovascular magnetic resonance imaging in patients recently recovered from coronavirus disease 2019 (COVID-19). JAMA Cardiol. 5, 1265-1273 (2020).

41. Rajpal, S. et al. Cardiovascular magnetic resonance findings in competitive athletes recovering from COVID-19 infection. JAMA Cardiol. 6, 116-118 (2021).

42. Moody, W. E. et al. Persisting adverse ventricular remodeling in COVID-19 survivors: a longitudinal echocardiographic study. J. Am. Soc. Echocardiogr. 34, 562-566 (2021).

43. Levi, M., Thachil, J., Iba, T. \& Levy, J. H. Coagulation abnormalities and thrombosis in patients with COVID-19. Lancet Haematol. 7, e438-e440 (2020).

44. Patell, R. et al. Postdischarge thrombosis and hemorrhage in patients with COVID-19. Blood 136, 1342-1346 (2020).

45. Leentjens, J., van Haaps, T. F., Wessels, P. F., Schutgens, R. E. G. \& Middeldorp, S. COVID-19-associated coagulopathy and antithrombotic agents-lessons after 1 year. Lancet Haematol. 8, e524-e533 (2021).

46. Suarez-Farinas, M. et al. Intestinal inflammation modulates the expression of ACE2 and TMPRSS2 and potentially overlaps with the pathogenesis of SARS-CoV-2-related disease. Gastroenterology 160, 287-301 e220 (2021).

47. Livanos, A. E. et al. Intestinal host response to SARS-CoV-2 infection and COVID-19 outcomes in patients with gastrointestinal symptoms. Gastroenterology 160, 2435-2450.e2434 (2021). 
48. Mao, R. et al. Manifestations and prognosis of gastrointestinal and liver involvement in patients with COVID-19: a systematic review and meta-analysis. Lancet Gastroenterol. Hepatol. 5, 667-678 (2020).

49. Weng, J. et al. Gastrointestinal sequelae 90 days after discharge for COVID-19. Lancet Gastroenterol. Hepatol. 6, 344-346 (2021).

50. Wu, Y. et al. Prolonged presence of SARS-CoV-2 viral RNA in faecal samples. Lancet Gastroenterol. Hepatol. 5, 434-435 (2020).

51. Gaebler, C. et al. Evolution of antibody immunity to SARS-CoV-2. Nature 591, 639-644 (2021).

52. Yang, J. K., Lin, S. S., Ji, X. J. \& Guo, L. M. Binding of SARS coronavirus to its receptor damages islets and causes acute diabetes. Acta Diabetol. 47, 193-199 (2010).

53. Rubino, F. et al. New-onset diabetes in Covid-19. N. Engl. J. Med 383, 789-790 (2020).

54. Montefusco, L. et al. Acute and long-term disruption of glycometabolic control after SARS-CoV-2 infection. Nat. Metab. 3, 774-785 (2021).

55. Brancatella, A. et al. Subacute thyroiditis after Sars-COV-2 infection. J. Clin. Endocrinol. Metab. 105, 2367-2370 (2020).

56. Tee, L. Y., Harjanto, S. \& Rosario, B. H. COVID-19 complicated by Hashimoto's thyroiditis. Singapore Med. J. 62, 265 (2020).

57. Mateu-Salat, M., Urgell, E. \& Chico, A. SARS-COV-2 as a trigger for autoimmune disease: report of two cases of Graves' disease after COVID-19. J. Endocrinol. Invest. 43, 1527-1528 (2020).

58. Robbins-Juarez, S. Y. et al. Outcomes for patients with COVID-19 and acute kidney injury: a systematic review and meta-analysis. Kidney Int. Rep. 5, 1149-1160 (2020)

59. Stevens, J. S. et al. High rate of renal recovery in survivors of COVID-19 associated acute renal failure requiring renal replacement therapy. PLoS ONE 15, e0244131 (2020).

60. Freeman, E. E. et al. The spectrum of COVID-19-associated dermatologic manifestations: an international registry of 716 patients from 31 countries J. Am. Acad. Dermatol. 83, 1118-1129 (2020).

61. Malkud, S. Telogen effluvium: a review. J. Clin. Diagn. Res. 9, WE01-WE03 (2015)

62. Abrantes, T. F. et al. Time of onset and duration of post-COVID-19 acute telogen effluvium. J. Am. Acad. Dermatol. 85, 975-976 (2021).

63. Bergamaschi, L. et al. Longitudinal analysis reveals that delayed bystander $\mathrm{CD}^{+} \mathrm{T}$ cell activation and early immune pathology distinguish severe COVID-19 from mild disease. Immunity 54, 1257-1275.e1258 (2021).

64. Hadjadj, J. et al. Impaired type I interferon activity and inflammatory responses in severe COVID-19 patients. Science 369, 718-724 (2020).

65. Vabret, N. et al. Immunology of COVID-19: current state of the science. Immunity 52, 910-941 (2020).

66. Phetsouphanh, C. et al. Immunological dysfunction persists for 8 months following initial mild-moderate SARS-CoV-2 infection. Nat. Immunol. https://doi.org/10.1038/s41590-021-01113-x (2022).

67. Hoeffel, G. et al. C-Myb ${ }^{+}$erythro-myeloid progenitor-derived fetal monocytes give rise to adult tissue-resident macrophages. Immunity 42, 665-678 (2015).

68. Bost, P. et al. Host-viral infection maps reveal signatures of severe COVID-19 patients. Cell 181, 1475-1488.e1412 (2020).

69. Schneider, C. et al. Induction of the nuclear receptor PPAR-gamma by the cytokine GM-CSF is critical for the differentiation of fetal monocytes into alveolar macrophages. Nat. Immunol. 15, 1026-1037 (2014).

70. Ziegler, C. G. K. et al. SARS-CoV-2 receptor ACE2 Is an interferon-stimulated gene in human airway epithelial cells and is detected in specific cell subsets across tissues. Cell 181, 1016-1035.e1019 (2020).

71. Hou, Y. J. et al. SARS-CoV-2 reverse genetics reveals a variable infection gradient in the respiratory tract. Cell 182, 429-446.e414 (2020).

72. Melms, J. C. et al. A molecular single-cell lung atlas of lethal COVID-19. Nature 595, 114-119 (2021).

73. Tsukui, T. et al. Collagen-producing lung cell atlas identifies multiple subsets with distinct localization and relevance to fibrosis. Nat. Commun. 11, 1920 (2020).

74. Coker, R. K. et al. Localisation of transforming growth factor betal and beta 3 mRNA transcripts in normal and fibrotic human lung. Thorax $\mathbf{5 6}$, 549-556 (2001).

75. Connors, J. M. \& Levy, J. H. COVID-19 and its implications for thrombosis and anticoagulation. Blood 135, 2033-2040 (2020).

76. Ackermann, M. et al. Pulmonary vascular endothelialitis, thrombosis, and angiogenesis in Covid-19. N. Engl. J. Med 383, 120-128 (2020).

77. Hottz, E. D. et al. Platelet activation and platelet-monocyte aggregate formation trigger tissue factor expression in patients with severe COVID-19. Blood 136, 1330-1341 (2020).

78. Ramlall, V. et al. Immune complement and coagulation dysfunction in adverse outcomes of SARS-CoV-2 infection. Nat. Med. 26, 1609-1615 (2020)

79. Libby, P. \& Luscher, T. COVID-19 is, in the end, an endothelial disease. Eur. Heart J. 41, 3038-3044 (2020).
80. South, K. et al. Preceding infection and risk of stroke: an old concept revived by the COVID-19 pandemic. Int. J. Stroke 15, 722-732 (2020).

81. Jhaveri, K. D. et al. Thrombotic microangiopathy in a patient with COVID-19. Kidney Int. 98, 509-512 (2020).

82. Gemayel, C., Pelliccia, A. \& Thompson, P. D. Arrhythmogenic right ventricular cardiomyopathy. J. Am. Coll. Cardiol. 38, 1773-1781 (2001).

83. Lazzerini, P. E., Laghi-Pasini, F., Boutjdir, M. \& Capecchi, P. L. Cardioimmunology of arrhythmias: the role of autoimmune and inflammatory cardiac channelopathies. Nat. Rev. Immunol. 19, 63-64 (2019).

84. Muccioli, L. et al. COVID-19-associated encephalopathy and cytokine-mediated neuroinflammation. Ann. Neurol. 88, 860-861 (2020).

85. Reichard, R. R. et al. Neuropathology of COVID-19: a spectrum of vascular and acute disseminated encephalomyelitis (ADEM)-like pathology. Acta Neuropathol. 140, 1-6 (2020).

86. Peleg, Y. et al. Acute kidney injury due to collapsing glomerulopathy following COVID-19 infection. Kidney Int. Rep. 5, 940-945 (2020).

87. Gentile, S., Strollo, F., Mambro, A. \& Ceriello, A. COVID-19, ketoacidosis and new-onset diabetes: are there possible cause and effect relationships among them? Diabetes Obes. Metab. 22, 2507-2508 (2020).

88. Salvio, G. et al. Bone metabolism in SARS-CoV-2 disease: possible osteoimmunology and gender implications. Clin. Rev. Bone Miner. Metab. 1, 1-7 (2020).

89. Wright, S. D., Tobias, P. S., Ulevitch, R. J. \& Ramos, R. A. Lipopolysaccharide (LPS) binding protein opsonizes LPS-bearing particles for recognition by a novel receptor on macrophages. J. Exp. Med. 170, 1231-1241 (1989).

90. Ravetch, J. V. \& Kinet, J. P. Fc receptors. Annu. Rev. Immunol. 9, 457-492 (1991).

91. Chakraborty, S. et al. Proinflammatory IgG Fc structures in patients with severe COVID-19. Nat. Immunol. 22, 67-73 (2021)

92. Barzilai, O., Ram, M. \& Shoenfeld, Y. Viral infection can induce the production of autoantibodies. Curr. Opin. Rheumatol. 19, 636-643 (2007).

93. Fujinami, R. S., von Herrath, M. G., Christen, U. \& Whitton, J. L. Molecular mimicry, bystander activation, or viral persistence: infections and autoimmune disease. Clin. Microbiol. Rev. 19, 80-94 (2006).

94. Ohashi, P. S. et al. Ablation of 'tolerance' and induction of diabetes by virus infection in viral antigen transgenic mice. Cell 65, 305-317 (1991).

95. Tuohy, V. K. et al. The epitope spreading cascade during progression of experimental autoimmune encephalomyelitis and multiple sclerosis. Immunol. Rev. 164, 93-100 (1998).

96. Zulfiqar, A. A., Lorenzo-Villalba, N., Hassler, P. \& Andres, E. Immune thrombocytopenic purpura in a patient with Covid-19. N. Engl. J. Med. 382, e43 (2020).

97. Toscano, G. et al. Guillain-Barre syndrome associated with SARS-CoV-2. N. Engl. J. Med 382, 2574-2576 (2020)

98. Gutierrez-Ortiz, C. et al. Miller Fisher syndrome and polyneuritis cranialis in COVID-19. Neurology 95, e601-e605 (2020).

99. Bowles, L. et al. Lupus anticoagulant and abnormal coagulation tests in patients with Covid-19. N. Engl. J. Med. 383, 288-290 (2020).

100. Rowley, A. H. Understanding SARS-CoV-2-related multisystem inflammatory syndrome in children. Nat. Rev. Immunol. 20, 453-454 (2020).

101. Bastard, P. et al. Autoantibodies against type I IFNs in patients with life-threatening COVID-19. Science 370, 423 (2020).

102. Chang, S. E. et al. New-onset IgG autoantibodies in hospitalized patients with COVID-19. Nat. Commun. 12, 5417 (2021).

103. Wang, E. Y. et al. Diverse functional autoantibodies in patients with COVID-19. Nature 595, 283-288 (2021)

104. LongCovidSOS. The impact of COVID vaccination on symptoms of long Covid. An international survey of 900 people with lived experience. https://www.pslhub.org/learn/coronavirus-covid19/data-and-statistics/theimpact-of-covid-vaccination-on-symptoms-of-long-covid-an-internationalsurvey-of-900-people-with-lived-experience-may-2021-r4636/ (2021).

105. Wolfel, R. et al. Virological assessment of hospitalized patients with COVID-2019. Nature 581, 465-469 (2020).

106. Sun, J. et al. Prolonged persistence of SARS-CoV-2 RNA in body fluids. Emerg. Infect. Dis. 26, 1834-1838 (2020).

107. Randall, R. E. \& Griffin, D. E. Within host RNA virus persistence: mechanisms and consequences. Curr. Opin. Virol. 23, 35-42 (2017).

108. Baigent, C., Burbury, K. \& Wheeler, D. Premature cardiovascular disease in chronic renal failure. Lancet 356, 147-152 (2000).

109. Ouchi, N., Parker, J. L., Lugus, J. J. \& Walsh, K. Adipokines in inflammation and metabolic disease. Nat. Rev. Immunol. 11, 85-97 (2011).

\section{Acknowledgements}

This work was supported by grant numbers U24 AI118644-05S1, P30 CA19652105S2, Fast Grant (George Mason University) and Gates Foundation (M.M.) and DK123749 0S1 (S.M.). 


\section{Competing interests}

M.M. and S.M. declare no conflicts of interest relating to this work.

\section{Additional information}

Correspondence should be addressed to Saurabh Mehandru or Miriam Merad.

Peer review information Nature Immunology thanks Akiko Iwasaki and the other, anonymous, reviewer(s) for their contribution to the peer review of this work.
Jamie D. K. Wilson, in collaboration with the Nature Immunology team, was the primary handling editor.

Reprints and permissions information is available at www.nature.com/ reprints.

Publisher's note Springer Nature remains neutral with regard to jurisdictional claims in published maps and institutional affiliations.

(c) Springer Nature America, Inc. 2022 